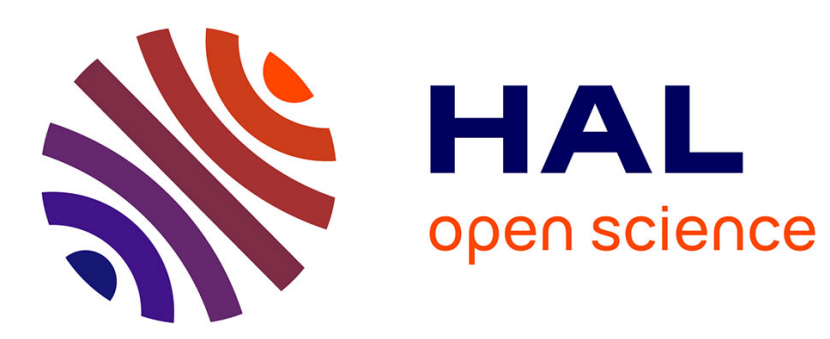

\title{
La représentation obligatoire des actionnaires salariés au conseil d'administration : un état des lieux
}

\author{
Xavier Hollandts, Nicolas Aubert
}

\section{To cite this version:}

Xavier Hollandts, Nicolas Aubert. La représentation obligatoire des actionnaires salariés au conseil d'administration: un état des lieux. Gestion 2000, 2011. halshs-01256778v2

\section{HAL Id: halshs-01256778 \\ https://shs.hal.science/halshs-01256778v2}

Submitted on 31 Dec 2018

HAL is a multi-disciplinary open access archive for the deposit and dissemination of scientific research documents, whether they are published or not. The documents may come from teaching and research institutions in France or abroad, or from public or private research centers.
L'archive ouverte pluridisciplinaire HAL, est destinée au dépôt et à la diffusion de documents scientifiques de niveau recherche, publiés ou non, émanant des établissements d'enseignement et de recherche français ou étrangers, des laboratoires publics ou privés. 


\title{
La représentation obligatoire des actionnaires salariés au conseil d'administration : un état des lieux
}

Xavier Hollandts, Professeur assistant, ESC Clermont, chercheur associé Institut Français de Gouvernement des Entreprises (IFGE) et CRCGM ${ }^{1}$.

xavier.hollandts@esc-clermont.fr

Nicolas Aubert, Professeur des Universités, Université du Sud Toulon-Var, Chercheur associé IFGE.

naubert@univ-tln.fr

\section{Résumé}

L'actionnariat salarié a connu un développement important depuis ces vingt dernières années. Les salariés constituent désormais des actionnaires de référence dans le capital des entreprises. Dans ce contexte, la loi du 30 décembre 2006 garantit désormais la représentation des actionnaires salariés au sein des conseils d'administration ou de surveillance des sociétés cotées. Partant de ce constat, cet article examine la question de recherche suivante : quelles sont les conséquences de la représentation des actionnaires salariés sur le fonctionnement du gouvernement d'entreprise? Cette représentation étant rendue obligatoire, nous avons souhaité réaliser un recensement exhaustif des représentants des actionnaires salariés avant l'entrée en vigueur de la loi afin de mieux connaître ce nouveau type d'administrateurs. Ce recensement nous a permis d'identifier le contexte dans lequel ces représentants évoluent mais également de mettre en évidence les caractéristiques personnelles et biographiques de ces représentants.

Mots-clés: Gouvernement d'entreprise, conseil d'administration, actionnariat salarié, représentation des salariés.

\begin{abstract}
Employee ownership experienced an important development for the last twenty years. In this context, employees become major shareholders. The 30 December 2006 bill makes the presence of a least one employee owner on the board of directors mandatory. From this point of view, this paper investigates one research question: what the representation of employee owners on the board brings to corporate governance? In this paper, we propose a thorough census of employee owner's representatives in order to identify the context of such representation and personal characteristics of board employee owner's representatives.

Keywords: Corporate governance, board of directors, employee ownership, employee representation.
\end{abstract}

\footnotetext{
${ }^{1}$ Auteur correspondant.
} 


\section{Introduction}

L'actionnariat salarié est un phénomène mondial qui connait désormais un important développement au point d'apparaître comme un élément majeur du capitalisme actionnarial [Aglietta et Rébérioux 2004]. Aux Etats-Unis, près d'un salarié sur quatre est aussi actionnaire salarié [NCEO 2006]. En France, cette proportion s'établit à environ un salarié sur dix [source: calculs des auteurs d'après les données FAS]. L'actionnariat salarié est en progression constante en France, puisqu'on dénombrait 700000 actionnaires salariés en 1998 contre près de 3 millions en 2006 [FAS 1998-2006].

L'actionnariat salarié a comme particularité d'être aussi bien apprécié par les gouvernements successifs que les directions des entreprises privées. Pour les premiers, l'actionnariat salarié permet de soulager les systèmes publics de financement des retraites en transférant une partie de cette charge sur la capitalisation individuelle des salariés. Pour les entreprises privées, l'actionnariat salarié offre des avantages indéniables en fidélisant et motivant les salariés tout en offrant l'avantage de mieux stabiliser le capital et de limiter l'impact des variations boursières sur la prise de décision stratégique [Desbrières 2002]. Dans ce contexte, les salariés constituent désormais des actionnaires de référence dans le capital des entreprises. Depuis 2002, une série de lois est venue consacrer la montée en puissance des actionnaires salariés en leur garantissant désormais au moins un poste d'administrateur (ou de membre de conseil de surveillance). En vertu de l'article 32 de la loi n ${ }^{\circ}$ 2006-1770 du 30 décembre 2006, les sociétés cotées ayant un actionnariat salarié supérieur à $3 \%$ du capital doivent obligatoirement faire élire un administrateur représentant les actionnaires salariés. L'application de cette loi porte à 41 le nombre d'entreprises du SBF 250 (16\% de l'indice) dans lesquelles les actionnaires salariés doivent être représentés au conseil d'administration ou de surveillance (au 31/12/2007).

Dans ce contexte de montée en puissance de l'actionnariat salarié, on peut s'interroger sur les conséquences de l'irruption des représentants des actionnaires salariés au sein des conseils d'administration français. En effet, la présence de représentants des actionnaires salariés est susceptible d'avoir un impact sur la fonction disciplinaire du conseil d'administration ou de surveillance. La littérature à ce sujet porte un regard relativement ambigu sur cette question : les représentants des actionnaires salariés peuvent aussi bien constituer un atout qu'un handicap pour le fonctionnement du conseil d'administration ou de surveillance. Dans cette optique, nous examinons, dans un premier temps, l'impact de la présence d'un représentant des actionnaires salariés sur l'efficacité du gouvernement de l'entreprise. Dans une seconde partie, nous présentons un état des lieux de la représentation des actionnaires salariés au conseil sur la période 2000-2005 : Qui sont-ils ? Quel est leur profil ? Dans quel type de conseil évoluent-ils? 


\section{La représentation des actionnaires salariés au gouvernement d'entreprise est-elle souhaitable?}

La représentation des actionnaires salariés peut théoriquement se justifier au regard du double investissement consenti par les actionnaires salariés. Ce double investissement incite très fortement les représentants des actionnaires salariés à exercer efficacement leur mandat d'administrateur (ou de membre du conseil de surveillance). Cependant, les dirigeants peuvent être tentés de détourner à leur profit les mécanismes d'actionnariat salarié et la représentation qui peut en découler, afin de faciliter leur enracinement.

\subsection{Les actionnaires salariés sont doublement incités à participer de manière efficace au gouvernement d'entreprise}

Le problème d'agence central du gouvernement d'entreprise consiste à contrôler le comportement et la performance des dirigeants, dans une perspective de maximisation de la valeur actionnariale [Charreaux 1997]. Dans ce contexte, les actionnaires salariés représentent des actionnaires singuliers qui ont la particularité de réaliser un investissement supplémentaire dans l'entreprise : l'investissement de leur capital humain [Desbrières 2002]. Les salariés assument donc un premier risque, qui est celui de leur investissement en capital humain. Visà-vis des autres actionnaires, ils ont une capacité de sortie beaucoup plus faible [Aglietta et Rebérioux 2004], le marché du travail étant moins liquide que le marché financier en raison de la faible redéployabilité du capital humain [Hansmann 1996 ; Blair 1999]. Les actionnaires salariés assument également un risque supplémentaire, lié à l'investissement de leur capital financier en titres de l'entreprise. Ce choix peut paraître curieux, étant donné qu'il ne répond pas aux critères d'optimalité d'allocation d'actifs en augmentant fortement le risque spécifique de chaque portefeuille [Ramaswamy 2003 ; Meulbroek 2005]. Ainsi, de toutes les parties prenantes, les actionnaires salariés représentent la catégorie qui assume le risque le plus élevé, bien supérieur à celui des actionnaires externes [Desbrières 2002]. Cette caractéristique essentielle, qui constitue la singularité de l'actionnariat salarié, est susceptible de légitimer la présence de représentants des actionnaires salariés au sein des instances de gouvernement d'entreprise.

Le double investissement consenti détermine directement l'utilité des actionnaires salariés à être représentés au sein du système de gouvernement [Desbrières 2002]. L'actionnariat salarié est ainsi un puissant vecteur d'alignement des intérêts sur l'objectif de création de valeur actionnariale [Pugh et alii 1999; Gamble 2000]. La présence d'un représentant des actionnaires salariés au gouvernement d'entreprise est globalement bénéfique pour les dirigeants et les actionnaires puisque les actionnaires salariés sont rationnellement favorables à la création de valeur actionnariale. De manière générale, les représentants des actionnaires salariés réduisent l'asymétrie informationnelle dont bénéficient classiquement les dirigeants vis-à-vis des actionnaires externes. Le contrôle et la surveillance des dirigeants par les représentants des actionnaires salariés seraient plus efficaces si ces derniers ont une proximité physique avec le top management [Gamble 2000]. Avec le soutien des représentants d'actionnaires externes, ils sont susceptibles de "prendre en étau» les dirigeants et de favoriser ainsi un contrôle efficace du comportement et des performances des dirigeants. En participant aux fonctions de ratification et de surveillance [Fama et Jensen 1983], ils sont en mesure d'exercer au mieux leur mandat (contrôler directement les comportements opportunistes des dirigeants) afin de maximiser la valeur totale de leur investissement (salaire + primes + valeur de leur portefeuille investi en actions de la société + dividendes). 
Cependant, les dirigeants peuvent également tenter de détourner à leur profit les mécanismes d'actionnariat salarié et la représentation qui en découle à leur avantage.

\subsection{Les risques de la représentation des actionnaires salariés au sein du gouvernement d'entreprise : l'enracinement des dirigeants}

Même si la représentation des actionnaires salariés peut sembler légitime et comporte certains avantages que nous venons de détailler, elle se heurte néanmoins à un risque opportuniste important représenté par l'enracinement des dirigeants. L'actionnariat salarié en tant que tel est susceptible d'offrir une opportunité supplémentaire d'enracinement pour les dirigeants, qui se trouvait renforcée en cas de représentation des actionnaires salariés au gouvernement d'entreprise.

La littérature souligne qu'outre les dispositions fiscales avantageuses [Scholes et Wolfson 1990 ; Autenne 2005], l'actionnariat salarié permet aux dirigeants d'établir un mécanisme défensif leur bénéficiant directement [Rauh 2006]. L'actionnariat salarié permet d'ériger des barrières aux prises de contrôle hostiles et donc de mieux protéger les dirigeants des risques d'éviction et de remplacement [Gordon et Pound 1990 ; Rauh 2006]. De manière générale, l'actionnariat salarié peut être considéré comme une fraction "amicale » de l'actionnariat pour les dirigeants [Chaplinsky et Niehaus 1994]. Cette technique anti-OPA peut d'ailleurs être considérée comme étant plus efficace que d'autres systèmes plus connus tels que les pilules empoisonnées, les parachutes dorés, les recapitalisations, les Leveraged Buy Out ou les investissements à long terme [Chaplinsky et Niehaus 1994].

Quand l'actionnariat salarié est couplé à une représentation au gouvernement d'entreprise, il offre un levier d'enracinement supplémentaire aux dirigeants [Gordon et Pound 1990 ; Pugh et alii 1999]. Les dirigeants, souvent accusés d'influencer la nomination des administrateurs [Mace 1971 ; Kosnik 1987 ; Wade et alii 1990], sont tentés de nommer des administrateurs qui leur sont favorables [Zajac et Westphal 1996], à l'instar des administrateurs représentant les actionnaires salariés. En effet, les dirigeants tablent sur le lien de dépendance hiérarchique existant entre eux et le représentant des actionnaires salariés. "L'alliance naturelle » existant entre salariés et dirigeants [Hellwig 2000] incitent les dirigeants à nouer des contrats implicites avec les actionnaires salariés [Pagano et Volpin 2005] en contrepartie d'un contrôle «amical» des actionnaires salariés [Gamble 2000]. Les dirigeants peuvent sécuriser leur propre position en envoyant par exemple des signaux positifs (sauvegarde de l'emploi, augmentation de salaires) en contrepartie d'un soutien des représentants des actionnaires salariés. En maintenant l'équipe dirigeante en place, les actionnaires salariés s'assurent que les contrats implicites seront bien exécutés. Il y a alors un risque de collusion et de protection mutuelle entre dirigeants et actionnaires salariés. A ce titre Faleye, Morck et Mehrotra [2006] évoquent un risque "d'enracinement bilatéral » où les actionnaires salariés s'enracinent tout comme les dirigeants, ce qui conduit les entreprises disposant d'un actionnariat salarié significatif à afficher notamment des performances plus faibles. Avant l'entrée en vigueur de la loi du 30 décembre 2006, nous avons souhaité réaliser un recensement exhaustif afin de mieux connaître ce nouveau type d'administrateurs appelés à être présents plus largement au sein des conseils d'administration ou de surveillance des entreprises cotées françaises. 


\section{La participation des actionnaires salariés au gouvernement de l'entreprise : un état des lieux}

Nous avons effectué un recensement des administrateurs représentant les actionnaires salariés au sein l'indice SBF 250 sur la période 2000-2005. Nous avons recueilli dans la mesure du possible un certains nombre de caractéristiques personnelles et d'éléments biographiques. Dans un premier temps, nous examinerons certaines caractéristiques des entreprises accueillant des représentants des actionnaires salariés. Puis nous mettrons en évidence un certain nombre de caractéristiques personnelles avant de dégager un portrait-type du représentant des actionnaires salariés.

\subsection{Le contexte de représentation des actionnaires salariés au sein des entreprises cotées françaises}

L'actionnariat salarié en France se diffuse de manière régulière dans la plupart des entreprises cotées. Certes, l'actionnariat salarié reste un phénomène cantonné aux grandes entreprises mais qui tend à se diffuser plus largement, y compris au sein des entreprises non cotées et/ou de taille plus modeste. En ce qui concerne les entreprises cotées, elles doivent faire obligatoirement état de la participation de leurs salariés au capital. Le tableau 1 fournit une vue d'ensemble de l'état de l'actionnariat salarié dans les entreprises cotées françaises. Trois phénomènes peuvent être relevés. Le premier est que l'on constate une augmentation régulière du nombre d'entreprises ayant un actionnariat salarié recensé. Aujourd'hui, plus d'une entreprise sur deux de l'indice SBF 250 dispose d'un actionnariat salarié. En revanche le taux moyen d'actionnariat salarié diminue sous l'effet de deux éléments : la stagnation du taux de capital détenu par les salariés dans les entreprises l'ayant déjà instauré (par absence de nouvelles opérations d'augmentation de capital réservées aux salariés) et le faible taux de capital détenu par les entreprises l'ayant récemment mis en place. Troisième phénomène, on constate un rattrapage entre le nombre d'entreprises disposant effectivement d'une représentation des actionnaires salariés et le nombre d'entreprises théoriquement soumises à cette représentation.

Tableau 1 : Evolution du taux d'actionnariat salarié et de l'état de la représentation des actionnaires salariés au sein du SBF 250 (2000-2005)

\begin{tabular}{|c|c|c|c|c|}
\hline Année & $\begin{array}{l}\text { Entreprises avec } \\
\text { actionnariat salarié } \\
(>0,01 \% \text { du capital })\end{array}$ & $\begin{array}{c}\text { Taux moyen } \\
\text { d'actionnariat } \\
\text { salarié }\end{array}$ & $\begin{array}{c}\text { Nombre } \\
\text { d'entreprise }>3 \% \\
\text { d'actionnariat } \\
\text { salarié }\end{array}$ & $\begin{array}{c}\text { Entreprise avec } \\
\text { représentation } \\
\text { des actionnaires } \\
\text { salariés } \\
\text { (au moins un } \\
\text { représentant }\end{array}$ \\
\hline 2000 & 121 & $2,96 \%$ & 32 & 9 \\
\hline 2001 & 125 & $3,06 \%$ & 35 & 12 \\
\hline 2002 & 127 & $3,02 \%$ & 39 & 15 \\
\hline 2003 & 129 & $2,92 \%$ & 42 & 17 \\
\hline 2004 & 132 & $2,77 \%$ & 37 & 20 \\
\hline 2005 & 134 & $2,64 \%$ & 37 & 21 \\
\hline
\end{tabular}

\footnotetext{
${ }^{2}$ Le dénombrement s'est effectué sur la base d'une variable binaire prenant la valeur de 1 si au moins un représentant des actionnaires salariés siège au conseil d'administration ou de surveillance ; sinon $=0$.
} 


\subsection{Le contexte de représentation des actionnaires salariés au sein des conseils}

Les représentants des actionnaires salariés évoluent avant tout au sein des très grandes entreprises cotées françaises, la plupart appartenant ou ayant appartenu au CAC $40^{3}$. Parmi ces entreprises, on peut citer Air-France, Alcatel, Areva, Axa, Bouygues, Credit Agricole, Eiffage, Essilor, France Telecom, Renault, Rhodia, Saint-Gobain, Schneider, Thales, Thomson, Total, Vinci mais également des sociétés de taille moins importante comme Boiron, CNP, Geodis, Latecoere, VM Matériaux. La conséquence directe est que les représentants des actionnaires salariés siègent au sein de conseils de grande taille, en moyenne d'un peu plus de 15 membres. Alors que les entreprises disposant d'un actionnariat salarié sans représentation au conseil ont des conseils légèrement supérieurs à 11 membres tandis que les entreprises ne disposant pas d'actionnariat salarié ont des conseils qui n'excèdent pas 9 membres.

Tableau 2 : Taille des conseils d'administration

\begin{tabular}{|c|c|c|c|}
\hline Année & $\begin{array}{c}\text { Nombre moyen } \\
\text { d'administrateurs } \\
\text { dans les entreprises } \\
\text { sans actionnariat } \\
\text { salarié }\end{array}$ & $\begin{array}{c}\text { Nombre moyen } \\
\text { d'administrateurs dans } \\
\text { les entreprises avec } \\
\text { actionnariat salarié }\end{array}$ & $\begin{array}{c}\text { Nombre moyen } \\
\text { administrateurs dans } \\
\text { les entreprises avec } \\
\text { représentation AS }\end{array}$ \\
\hline 2000 & 8.39 & 11.25 & $15.11^{* * *}$ \\
\hline 2001 & 8.74 & 11.27 & $16.16^{* * *}$ \\
\hline 2002 & 8.63 & 11.42 & $16.2^{* * * *}$ \\
\hline 2003 & 8.91 & 11.55 & $15.41^{* * *}$ \\
\hline 2004 & 9.02 & 11.59 & $15.5^{* * *}$ \\
\hline 2005 & 9.07 & 11.55 & $15.14^{* * *}$ \\
\hline
\end{tabular}

Par ailleurs, les représentants des actionnaires salariés évoluent au sein de conseil d'administration ou de surveillance qui présentent des caractéristiques spécifiques. Ainsi, ils évoluent au sein de conseils qui se caractérisent par la proportion plus élevée d'administrateurs « indépendants » (cf tableau 3).

\footnotetext{
${ }^{3}$ Les entreprises disposant d'une représentation des actionnaires salariés affichent un chiffre d'affaires moyen de 18 milliards d'euros alors que les autres entreprises de l'échantillon n'ayant pas d'actionnariat salarié affichent un chiffre d'affaires moyen d' 1,5 milliards d'euros.

${ }^{4}$ Nous avons réalisé un test de différence de moyenne afin de comparer les deux sous-échantillons (entreprise avec et sans actionnariat salarié). Les tests sont tous positifs et significatifs au seuil de $1 \%$. Idem pour le tableau 3.
} 
Tableau 3 : Proportion d'administrateurs indépendants suivant le type de conseil

\begin{tabular}{|c|c|c|}
\hline Année & $\begin{array}{c}\text { Proportion d'administrateurs } \\
\text { indépendants dans les conseils des } \\
\text { entreprises avec représentation AS }\end{array}$ & $\begin{array}{c}\text { Proportion d'administrateurs } \\
\text { indépendants reste SBF 250 }\end{array}$ \\
\hline 2000 & $52 \%$ & $40 \%$ \\
\hline 2001 & $56 \%$ & $42 \%$ \\
\hline 2002 & $55 \%$ & $45 \%$ \\
\hline 2003 & $60 \%$ & $53 \%$ \\
\hline 2004 & $66 \%$ & $59 \%$ \\
\hline 2005 & $62 \%$ & $57 \%$ \\
\hline Moyenne & $59 \% * *$ & $49 \%$ \\
\hline
\end{tabular}

Les représentants des actionnaires salariés siègent au sein de conseils où le PDG affiche un âge plus élevé en moyenne (58 ans) que le reste de l'échantillon (54 ans ; moyenne SBF 250 : 54,5 ans) mais ont une ancienneté inférieure à 9 ans (reste de l'échantillon: 11,8 ans ; moyenne SBF $250: 11,5$ ans). Dans seulement $6 \%$ des cas, ils font face à un PDG fondateur alors que les PDG-fondateurs représentent 25\% du SBF 250 sur la période. Par ailleurs, ils siègent au sein de conseils où le PDG ne détient qu'une faible part du capital $(1,15 \%$ en moyenne) alors que cette part est sensiblement plus élevée dans le reste du SBF 250 $(15,85 \%)$. Ainsi, les représentants des actionnaires salariés siègent avant tout dans de grandes entreprises. Ces entreprises ont comme caractéristiques de ne pas être des entreprises familiales (hormis Boiron, Bouygues et VM Matériaux) et d'avoir un capital moins concentré ${ }^{5}$ que les autres entreprises de l'échantillon. Par ailleurs, les représentants des actionnaires salariés siègent au sein de conseil de taille importante, comportant une proportion plus élevée d'administrateurs indépendants et dans lesquels le PDG devrait théoriquement moins être en mesure de s'enraciner (ancienneté plus faible et part du capital détenu relativement faible ; ces deux dimensions sont généralement mises en avant dans la littérature pour expliquer la faculté d'un dirigeant exécutif à se maintenir à son poste, autrement dit s'enraciner - voir Shen (2003) pour des précisions).

Au sein de ces conseils, la présence de représentants des actionnaires salarié est généralement limitée à une seule personne, rarement deux. Ainsi la moyenne de représentant d'actionnaires salariés par conseil s'établit à 1,3. En ce qui concerne les quelques éléments biographiques dont nous disposons dans les rapports annuels ou les documents de référence AMF, il ressort que le représentant des actionnaires salariés est âgé en moyenne de 51 ans. Son ancienneté moyenne excède rarement 3 ans ce qui indique qu'il exerce rarement 2 mandats consécutifs. Enfin, il détient en moyenne environ 2000 actions, ce chiffre étant d'ailleurs en augmentation sensible au cours des dernières années notamment sous l'effet des levées d'options concernant certains représentants des actionnaires salariés issus des la population cadre.

\footnotetext{
5 La somme des cinq premiers actionnaires, proxy classique de mesure de la concentration actionnariale, est de $40 \%$ pour les entreprises disposant d'une représentation des actionnaires salariés contre plus de $52 \%$ pour le reste du SBF 250.
} 
Tableau 4 : Caractéristiques personnelles moyennes des représentants des actionnaires salariés

\begin{tabular}{|c|c|c|c|c|c|}
\hline Année & $\begin{array}{c}\text { Nombre } \\
\text { d'entreprises avec } \\
\text { représentation des } \\
\text { actionnaires } \\
\text { salariés }\end{array}$ & $\begin{array}{c}\text { Nombre total } \\
\text { d'administrateurs } \\
\text { représentant les } \\
\text { AS }\end{array}$ & Age moyen & $\begin{array}{c}\text { Ancienneté } \\
\text { moyenne en } \\
\text { poste }\end{array}$ & $\begin{array}{c}\text { Nombre } \\
\text { moyen } \\
\text { d'actions } \\
\text { détenues }\end{array}$ \\
\hline 2000 & 9 & 12 & 49.25 & 2.28 & 204 \\
\hline 2001 & 12 & 16 & 52.36 & 2.83 & 557 \\
\hline 2002 & 15 & 20 & 53.2 & 3.4 & 1990 \\
\hline 2003 & 17 & 22 & 52.63 & 3.41 & 2196 \\
\hline 2004 & 20 & 26 & 52.65 & 3.16 & 3169 \\
\hline 2005 & 21 & 25 & 51.68 & 2.2 & 5813 \\
\hline $\begin{array}{c}\text { Moyenne sur } \\
\text { la période }\end{array}$ & 15.66 & 20.16 & 51 ans & 3,1 ans & 1931,76 \\
\hline
\end{tabular}

\section{Encadré : Portrait-robot d'un représentant des actionnaires salariés}

Le représentant des actionnaires salariés au conseil (d'administration ou de surveillance) est avant tout.... un homme. Lors de notre recensement nous n'avons dénombré la présence que d'une femme chargée de représenter les actionnaires salariés chez Thales. Comme nous l'enseigne le tableau $\mathrm{n}^{\circ} 4$, le représentant des actionnaires salariés est souvent âgé d'un peu plus de 50 ans. Par conséquent, le représentant des actionnaires salariés exerce très rarement plus de deux mandats d'administrateurs sous l'effet notamment de la proximité de l'âge de la retraite lors de la fin de son premier mandat. Les représentants des actionnaires salariés peuvent parvenir à cette fonction par deux voies différentes: ils peuvent être élus par l'ensemble des actionnaires salariés lors d'une élection interne ou bien être présentés par l'association interne d'actionnaires salariés à l'assemblée générale des actionnaires après validation par la Direction Générale (pour plus de précisions, se reporter à Alanche [2007]). Parmi l'ensemble des entreprises ayant au moins un représentant des actionnaires salariés, quasiment toutes à l'exception de Renault comptaient un représentant issu de l'association interne d'actionnaires salariés, alors que les représentants syndicaux sont parfois tentés de gagner un poste supplémentaire d'administrateurs par ce biais-là [Alanche 2007]. En termes de catégories socio-professionnelles, les représentants des actionnaires salariés sont très majoritairement issus de la population cadre. Ainsi parmi les représentants des actionnaires salariés, on trouve par exemple cinq ingénieurs, un directeur général de filiale, deux contrôleurs de gestion, un directeur des investissements, un responsable des relations investisseurs, deux chargés de mission, deux responsables marketing, un juriste, un commandant de bord, un directeur financier, un responsable formation et gestion des compétences. Les représentants des actionnaires salariés détiennent en moyenne 1931 actions mais ce chiffre masque de grandes disparités puisque l'écart-type est de 1090, le minimum étant de 10 et le maximum de 55403. On peut tout de même noter une tendance assez nette à l'augmentation moyenne du nombre d'actions possédées par les représentants des actionnaires salariés (5813 actions par représentant en moyenne en 2005). 


\section{Conclusion}

Cet article a pour objectif de mettre en évidence les enjeux de la participation des actionnaires salariés au gouvernement d'entreprise. Ainsi, la participation des actionnaires salariés semble légitime mais se heurte à la question de son efficacité au regard des risques d'enracinement accrus. La loi du 30 décembre 2006 ne laisse plus la possibilité aux sociétés cotées de s'interroger sur l'intérêt d'une telle représentation, elle l'impose. La représentation des actionnaires salariés étant désormais acquise, il ne convient plus simplement de s'interroger sur son intérêt mais il convient de prolonger cette problématique en s'interrogeant sur les conditions pouvant garantir l'efficacité d'une telle représentation. Les représentants des actionnaires salariés sont théoriquement dotés des incitations nécessaires pour exercer correctement la fonction disciplinaire qui leur est dévolue. Par ailleurs, et même si quasiment aucune recherche n'a abordé la question de leurs apports aux travaux du conseil, les représentants des actionnaires salariés sont porteurs d'une connaissance approfondie des rouages de l'entreprise, de son climat social et de son histoire [IFGE 2005]. Néanmoins, les représentants des actionnaires salariés en raison de leur non-appartenance au monde syndical et de leur statut cadre semblent avoir une proximité «naturelle » avec leurs dirigeants [Pagano et Volpin 2005], ce qui accroît d'autant les risques de collusion et de protection mutuelle [Faleye et alii 2006]. Ainsi, la question de la légitimité et de l'efficacité des représentants des actionnaires salariés reste encore débattue. De ce point de vue le constat réalisé par Balligand et De Foucauld en 2000, semblait largement prémonitoire: «Le développement de la présence de salariés actionnaires dans les organes de gestion des entreprises ne passe pas seulement par des éléments objectifs - développement du nombre de salariés actionnaires mais requiert également que les salariés actionnaires prennent conscience de leur capacité à peser sur les décisions stratégiques de l'entreprise » [Balligand-De Foucauld 2000, p91].

Le recensement que nous avons effectué sur les entreprises cotées avant l'entrée en vigueur de la loi du 30 décembre 2006 met en évidence plusieurs éléments. A mesure que l'actionnariat salarié se diffuse, la représentation des actionnaires salariés tend à s'accroître. Cependant, cette représentation reste cantonnée au monde des «très grandes entreprises » qui ont les moyens et la volonté d'associer leurs salariés au capital et parfois à la gouvernance de leur organisation. Les représentants des actionnaires salariés siègent donc en grande majorité dans les entreprises appartenant ou ayant appartenu au CAC 40. Ils siègent dans des conseils de grande taille comportant une part majoritaire d'administrateurs indépendants. Les représentants des actionnaires sont quasiment tous issus de la population cadre et représentent des associations internes d'actionnaires salariés non issues du monde syndical. Ils accèdent à ce poste en fin de carrière et exercent rarement plus de deux mandats.

Au final, il reste ainsi aux actionnaires salariés à désormais saisir l'opportunité qui leur est offerte en participant pleinement au gouvernement de leur entreprise. Il reste néanmoins à mieux définir le rôle et la posture de ces administrateurs si particuliers, soumis aux mêmes obligations que n'importe quel administrateur, mais défenseurs d'un actionnariat interne et porteurs des valeurs historiques de l'entreprise. 


\section{Bibliographie}

Aglietta M., Reberioux A., (2004), Dérives du capitalisme financier, Albin Michel Economie, Paris. Alanche P., 2007, Renault Côté Cour : un salarié au conseil d'administration, Editions de l'Atelier, Paris.

Autenne A., (2005), Analyse économique du droit de l'actionnariat salarié, Bibliothèque de la Faculté de Droit de l'Université Catholique de Louvain, Bruylant, Bruxelles.

Balligand J-P., De Foucauld J-B., (2000), Rapport au Premier ministre sur l'épargne salariale, 27 janvier 2000.

Blair M., (1999), "Firm-specific human capital and theories of the firm », in Employees and Corporate Governance, Blair M. et Roe M. (eds.), Brooking Institution Press, Washington.

Chaplinsky S, Niehaus G., (1994), « The Role of ESOPs in Takeover Contests », Journal of Finance, vol.49, n 4 , p. 1451-1470.

Charreaux G., (1997), «Vers une théorie du gouvernement des entreprises, in Le Gouvernement des Entreprises, Charreaux G. (ed.), coll. Recherche en Gestion, Economica p. 421-469.

Desbrières Ph., (1997), «Le rôle de l'actionnariat des salariés non-dirigeants dans le système de gouvernement de l'entreprise » in Le Gouvernement des Entreprises, Charreaux G. (ed.), coll. Recherche en Gestion, Economica.

Desbrières Ph., (2002), «Les actionnaires salariés », Revue Française de Gestion, vol.28, n¹41, p. 255-281.

FAS Fédération Française des Actionnaires Salariés et Anciens Salariés, (2006), Guide de l'actionnaire salarié, $6^{\mathrm{e}}$ édition.

Faleye O., Mehrotra V., Morck R., (2006), «When Labor Has a Voice in Corporate Governance », Journal of Financial and Quantitative Analysis, vol.41, n³, p. 489-510.

Fama E. et Jensen M., (1983), "Separation of Ownership and Control », Journal of Law and Economics, vol.26, p. 301-326.

Gamble J., (2000), « Management Commitment to Innovation and ESOP Stock Concentration », Journal of Business Venturing, vol.15, p. 433-447.

Gordon L., Pound J. (1990), « ESOPs and Corporate Control, Journal of Financial Economics, vol. 27 , p. 525-555.

Hansmann H., (1996), The Ownership of Enterprise, Belknap Press of Harvard University Press.

Hellwig M., (2000), « On the economics and politics of corporate finance and corporate control», in Xavier Vives, ed.: Corporate Governance: Theoretical and Empirical Perspectives, Cambridge University Press, Cambridge, UK.

IFGE (Institut Français de Gouvernement des Entreprises/EM Lyon), 2005, La présence d'administrateurs salariés au conseil d'administration, série Cahier pour la Réforme, Avril.

Kosnik R., (1987), " Greenmail: A study of board performance in corporate governance ", Administrative Science Quarterly. vol.32, p. 163-185.

Mace M., (1971), Directors: Myth and Reality, Harvard University Graduate School of Business Administration, Boston.

Meulbroek L., (2005), « Company Stock in Pension Plans: How Costly Is It ? », Journal of Law and Economics, vol. 48, n $^{\circ} 2$, p. 443-474.

NCEO, (2006), données disponibles sur http://www.nceo.org/library/eo_stat.html

Pagano H., Volpin P., 2005, « Managers, Workers and Corporate Control », Journal of Finance, vol.60, n², p. 841-868.

Pugh W, Jahera J, Oswald S., (1999), «ESOPs, Takeover Protection, and Corporate DecisionMaking », Journal of Economics and Finance, vol.23, n², p.170-185.

Rauh J., (2006), Own Company in Defined Contribution Pension Plans: A takeover Defense ? », Journal of Financial Economics, vol.81, n² 2, p. 379-410.

Scholes M., Wolfson M., (1990), «Employee stock ownership plans and corporate restructuring: myths and realities », Financial Management, vol.19, p. 12-28.

Shen W., 2003, The dynamics of board-CEO relationship: An evolutionary perspective, Academy of Management Review, vol.28, ${ }^{\circ} 3$, pp 466-476. 